\title{
Diversity and distribution of avian haemosporidians in sub-Saharan Africa: an inter-regional biogeographic overview
}

\author{
DIANA C. OUTLAW ${ }^{1}$, JOHANNA A. HARVEY ${ }^{2}$, SERGEI V. DROVETSKI ${ }^{3}$ and \\ GARY VOELKER ${ }^{2}$ \\ ${ }^{1}$ Department of Biological Sciences, Mississippi State University, PO Box GY, Mississippi State, MS 39762, USA \\ ${ }^{2}$ Department of Wildlife and Fisheries Sciences, Texas A $\varpi M$ University, 210 Nagle, MS 2258, College Station, TX \\ 77843, USA \\ ${ }^{3}$ Division of Birds, National Museum of Natural History, Smithsonian Institution, 1000 Constitution Ave, NW, \\ Washington, DC 20004, USA
}

(Received 7 Fuly 2016; revised 7 October 2016; accepted 7 October 2016; first published online 8 November 2016)

SUMMARY

The diversity of avian malaria parasites is much greater than 20th century morphologists realized and virtually every study in this field in the last 15 years has uncovered previously undocumented diversity at multiple levels within the taxonomic hierarchy. Despite this explosion of knowledge, there remain vast sampling gaps, both geographically and host-taxonomically, which makes characterizing patterns of diversity extremely challenging. Here, we summarize the current state of knowledge of sub-Saharan African avian malaria parasite diversity, focusing on avian hosts endemic to Africa. The relative proportions of the parasite genera included here, Plasmodium, Haemoproteus (including Parahaemoproteus) and Leucocytozoon, varied between regions, in part due to habitat preferences of the insect vectors of these genera, and in part we believe due to sampling bias. Biogeographic regions of sub-Saharan Africa harbour about the same proportion of endemic to shared parasite lineages, but there appears to be no phylogenetic structuring across regions. Our results highlight the sampling problem that must be addressed if we are to have a detailed understanding of parasite diversity in Africa. Without broad sampling within and across regions and hosts, using both molecular tools and microscopy, conclusions about parasite diversity, host-parasite interactions or even transmission dynamics remain extremely limited.

Key words: Haemosporida, Africa, birds, malaria.

\section{INTRODUCTION}

The ecosystems of the African continent collectively harbour some of the highest continental bird diversity in the world; only the Neotropics and tropical Asia are more diverse. Not surprisingly, the malaria parasites of these birds appear to be as diverse as their avian hosts (see also Clark et al. 2014), as reflected in a number of regional studies conducted over the past decade (e.g. Smith et al. 2011; Loiseau et al. 2012; Okanga et al. 2014; Lauron et al. 2015; Lutz et al. 2015). While this diversity is to some extent driven by regional ecosystem differences including host distributions (see also Lauron et al. 2015), the underlying method of transmission is likely also a factor (Santiago-Alarcon et al. 2012). Although all three major genera of avian malaria parasites are vectored by dipteran arthropods, the current understanding is that Plasmodium is vectored by mosquitoes, whereas Haemoproteus is transmitted by louse flies or biting midges, and Leucocytozoon is transmitted by black flies

* Corresponding author: Department of Biological Sciences, Mississippi State University, PO Box GY, Mississippi State, MS 39762, USA. E-mail: doutlaw@ biology.msstate.edu
(Valkiūnas, 2005). The different ecological requirements of these dipteran vectors (largely related to water availability, water flow and temperature) play a major role in the overall distribution of each haemosporidian genus. Our goal here is to use existing genetic data on parasite lineages to provide a synthesis of known malaria parasite diversity across sub-Saharan Africa. To accomplish this, we have characterized patterns of diversity in African avian malaria parasites to address three basic questions: (1) How diverse are malaria parasites within the biogeographic regions of Africa?, (2) How are parasite lineages distributed geographically, i.e. what are the proportions of endemic parasites in each region and are some regions more likely to share lineages than others?, and (3) What are the host preferences and host breadths of these parasites?

\section{The state of the continent}

From very early microscopy analyses of $\sim 11500$ blood smears, 70 morphological Haemoproteus (including Parahaemoproteus and Haemoproteus) and 13 morphological Plasmodium species have been found in birds in Africa (Garnham, 1950; see Valkiūnas, 2005 for summary). Yet of these, just 
15 Parahaemoproteus, 2 Haemoproteus and 2 Plasmodium morpho-species were endemic to Africa. From the 1970 s through early 1990s, surveys based on microscopy of blood smears collected from a taxonomically broad spectrum of host species were conducted in sub-Saharan Africa, and prevalence (percentage of birds with detectable parasites in the blood) ranged from similar levels of $11.5 \%$ in Senegal, $13 \%$ in Cameroon and $19 \cdot 1 \%$ across subSaharan Africa (collectively), to a comparatively high level of $37 \%$ from East African savannah regions (Bennett and Herman, 1976; Bennett et al. 1978; Kirkpatrick and Smith, 1988; Bennett et al. 1992). In 2005, two very detailed studies, one focused on western African rainforests (Sehgal et al. 2005) and one from Uganda (Valkiūnas et al. 2005), highlighted the diversity of haemosporidian parasites in Africa using microscopy techniques. Combined, these two studies included 1276 individual birds, and haemosporidian parasites were identified in 27 avian families, with prevalence ranging from $28 \cdot 6$ to $61.9 \%$, with the highest numbers from Uganda.

Surveys of molecular phylogenetic relationships generally return higher diversity and prevalence estimates than microscopy, although these can be inflated due to circulating sporozoites. A recent survey of parasites in Western Congolian rainforests (Møller et al. 2011) identified haemosporidians from 25 host families from just 527 individual birds; Plasmodium infections alone were detected in $45 \%$ of individuals (Beadell et al. 2009). And indeed, most molecular examinations of parasite diversity in birds have focused on just a few families (primarily sunbirds [Nectariniidae] and bulbuls [Pycnonotidae]) from the Western African or Western Congolian rainforests (Bonneaud et al. 2009; Chasar et al. 2009; Loiseau et al. 2010; Iezhova et al. 2011; but see Hellgren et al. 2007 and Lauron et al. 2015). Recent exceptions to these taxonomically limited, rainforest-based studies are from the Vwaza Marsh in Malawi (Lutz et al. 2015) and wetlands in the Western Cape of South Africa (Okanga et al. 2014). The only study to assess diversity in the Saharan region used targeted sampling in northeastern Nigeria for a limited number of avian species $(n=9)$ thereby not reflecting the overall malaria diversity of that region (Waldenström et al. 2002).

In a broader study of global parasite diversity, Clark et al. (2014) include sub-Saharan Africa as a biodiversity hotspot for haemosporidian parasites, but do not explicitly discuss parasite distributions on the continent. In another study focusing on Plasmodium parasites from African sunbirds, Lauron et al. (2015) show that there is a great deal of variation in the distributions of Plasmodium lineages, with some being geographically limited and others very widespread. Likewise, some
Plasmodium lineages from sunbirds are probably more specialized on hosts than others.

\section{State of the sampling: limitations}

Studies of haemosporidian diversity in Africa, like those in virtually every other part of the world, have been inconsistent with collection and with laboratory protocols to detect parasites. Much of the variation that we see across Africa may be in part due to these research biases. The overwhelming majority of studies have focused on Haemoproteus and Plasmodium (Ricklefs and Fallon, 2002; Waldenström et al. 2002; Durrant et al. 2007; Pérez-Tris et al. 2007; Beadell et al. 2009; Bonneaud et al. 2009; Ishtiaq et al. 2012; Karamba et al. 2012), and sometimes only on Plasmodium (Beadell et al. 2006; Valkiūnas et al. 2009; Loiseau et al. 2012). Of the 15 studies, the data for which are included in our analyses, only three include data from Leucocytozoon (Hellgren et al. 2007; Ishak et al. 2008; Lutz et al. 2015), because these other studies did not look for this parasite. Further restricting the potential diversity is that most studies include only passerines (songbirds, representing roughly $1 / 2$ of avian diversity), or at least the majority of the samples came from passerines. And, finally, most studies have been geographically limited, as we discuss above.

We point out also that previous studies of African malaria parasites (see above) did not systematically sample across parasite lineages in their molecular techniques. In particular, primer bias issues in commonly used polymerase chain reaction (PCR) techniques exist such that some primers target primarily Plasmodium or Plasmodium and Haemoproteus but are not well suited for Leucocytozoon. Thus, any regional differences could be partially attributable to primer bias, rather than differences related to vectors and their life histories or to host specialization.

\section{Biogeographic regional parasite diversity}

Linder et al. (2012) developed statistical models of faunal distributions across sub-Saharan Africa and here we use that which they developed and defined for birds (i.e. the hosts). Virtually all African avifauna cluster into these seven statistically-defined sub-Saharan biogeographic regions (rather than geographic as in Lauron et al. 2015): Saharan (to include Mauritanian), Sudanian, Ethiopian, Somalian, Congolian, Zambezian and Southern African. Although each region does have some number of endemic bird species, areas with a larger proportion of endemics are the Ethiopian, Congolian and Zambezian regions; these three regions also have the highest levels of species richness (Jetz and Rahbek, 2002; Fjeldså, 2003). For the Ethiopian 
region, endemism and species richness are tied to distinctive high elevation mountains, whereas endemism and species richness in the Congolian region are tied to lowland tropical forest habitat. And, in the Zambezian region, endemism and species richness are largely associated with Afromontane forests in the East Arc Mountains of Tanzania and Kenya. Much of the remainder of the Zambezian region comprises semi-arid savannah and deciduous dry forests, and as such, much of the avifauna found in the Zambezian region is also found in the different and varying habitats found in the Southern African region as well (see Sinclair and Ryan, 2010 for avian distributions).

Similarly, the desert and semi-arid northern savannahs found in the Saharan, Sudanian and to some extent the Ethiopian and Somalian regions also tend to share avian species. The Sudanian region is unique however in hosting a significant number of wintering European migrant species (see Sinclair and Ryan, 2010). And finally, avifaunal assemblages to the north of the Sahara, for example those found in the Atlas Mountains of Morocco and Algeria, tend to have closer ties to the European avifauna than to sub-Saharan ones. As a broad generalization then, avian species tend to be restricted to one or more northern regions, the Congolian region, or to either or both of the southern regions (Zambezian and Southern African). Because of the regional variation in avian assemblages, we sought to evaluate whether these partitions are also apparent in avian malaria parasites.

\section{Geographic distribution of parasite lineages}

With the large number of endemic bird species within each of the biogeographic regions, we were interested in evaluating whether this regional variation is also reflected in their malaria parasites. Here, we define an endemic parasite by whether the lineage was only recovered from one region but was found in two or more host individuals. We also wanted to know how many lineages are shared between regions, i.e. whether a parasite lineage was found in two or more regions. Because the genera of malaria parasites use different insect vectors (see above), we also evaluated the proportions of each parasite genus within each region. For an evolutionary context, we conducted a phylogenetic analysis of all lineages to determine whether phylogenetic structure was linked to biogeographic regions in any way, i.e. do one or more regions harbour distinct clades of malaria parasites as you might expect if parasites were host specific?

\section{Host preferences}

Recent research has shown that there may be as many avian malaria parasite species as there are avian hosts (see Ricklefs et al. 2014), so parasites should be the most diverse in regions with the greatest proportions of endemic host species. To address this, we determined the number of host species in which each parasite lineage was detected and how host families are parasitized by genus. Five of the best sampled passerine families (Cisticolidae, Estrildidae, Nectariniidae, Ploceidae and Pycnonotidae) have their greatest diversity in Africa. However, because these families are widespread across Africa, their distributions may homogenize parasite communities across African regions to some extent due to the AbundanceOccupancy Relationship (Drovetski et al. 2014). Finally, we used the Host Specificity Index $\left(S_{\mathrm{TD}}\right.$; Poulin and Mouillot, 2003) to determine if, at the broad scale of genera (due to the limitations of 'positive-only' data in MalAvi; Bensch et al. 2009), parasite genera demonstrate different strategies of parasitism. Without prevalence data by species and region, we cannot eliminate the possibility of spillover infections into non-preferred or dead-end hosts confounding potential patterns; this is a major limitation of these data included here.

\section{MATERIALS AND METHODS}

\section{Data compilation}

We downloaded all malaria lineage data from MalAvi (April 2015; http://mbio-serv2.mbioekol. lu.se/Malavi/) and pruned the dataset to include only those lineages sampled from resident birds from continental sub-Saharan Africa (1068 cytochrome $b$ sequences in 410 lineages [unique sequences]). Limiting our sampling to sub-Saharan Africa allowed us to make use of the statisticallyderived African bird faunal regions from Linder et al. (2012) for inter-regional comparisons and assessments of regional endemism. We assigned each malaria lineage to one or more of four regions: Congolian, South African, Sudanian and Zambezian (Table 1). Somalian and Ethiopian regions were excluded from the analyses because no data from MalAvi exist from these regions, and Saharan samples were excluded because just 13 parasite sequences have been found there (i.e. too few for meaningful comparisons). For some analyses, we also excluded South African samples because only ten lineages were found there.

\section{Data analyses}

We compiled the numbers of lineages that were found in only one region (hereafter, 'endemic') or were found in two, or more regions (hereafter, 'shared'), but excluded lineages found only once (i.e. lineages represented by only one sequence) (Table 2). Using a general linear model (SPSS 
Table 1 . Lineages found only within one region, by genus $(P=0 \cdot 0002)$.

\begin{tabular}{lcccc}
\hline \hline Region/genus & Haemoproteus & Plasmodium & Leucocytozoon & Total \\
\hline Congolian & 8 & 19 & 0 & 27 \\
Sudanian & 6 & 4 & 7 & 17 \\
Zambezian & 15 & 11 & 19 & 45 \\
\hline \hline
\end{tabular}

Table 2. Number of lineages in each region that are endemic to that region and number of lineages found in each region that are shared with other (1 or more) regions $(P=0 \cdot 0617)$.

\begin{tabular}{lll}
\hline \hline Region & Endemic & Shared \\
\hline Congolian & 27 & 27 \\
Sudanian & 17 & 31 \\
Zambezian & 45 & 34 \\
\hline \hline
\end{tabular}

v24), we tested whether biogeographic regions varied in their proportions of hosts infected by parasite genera (Haemoproteus, Plasmodium and Leucocytozoon). Using a chi-square test (vassarstats.net), we calculated whether biogeographic regions differed in their proportions of endemic and shared lineages. Host specificity was measured using taxonomic distance between host species infected with the same malaria lineage by using the host specificity index $S_{\mathrm{TD}}$ and $\operatorname{Var} S_{\mathrm{TD}}$, which measures taxonomic evenness (Clarke and Warwick, 2001; Poulin and Mouillot, 2003). Host species are arranged into six broadly accepted taxonomic levels using class, superorder, order, family, genera and species (Dickinson and Remsen, 2013). These taxonomic steps provide the maximum value of $S_{\mathrm{TD}}$ where 5 is the maximum steps needed to reach a common ancestor (Class Aves) when all other taxonomic classes are different and 1 being the minimum value when host are sister species; lower specificity values indicate increased host specificity. On the other hand, $\operatorname{Var} S_{\mathrm{TD}}$ values are indicative of symmetry in taxonomic structure (or evenness), such that a low score would indicate equal taxonomic distances (steps in the taxonomic hierarchy employed) across host species, whereas high values would indicate taxonomic asymmetry or unequal taxonomic distances across host species. Parasite lineages recovered once were removed from $S_{\mathrm{TD}}$ and $\operatorname{Var} S_{\mathrm{TD}}$ analyses as no comparisons can be made.

\section{Phylogenetic analysis}

We reconstructed 10000000 trees of all lineages using a Bayesian framework (BEAST v1.6.2 with $\mathrm{HKY}+\mathrm{I}+\boldsymbol{\Gamma}$ model of nucleotide substitution with estimated nucleotide frequencies and a Yule Process [speciation]) sampling every 1000 trees
(Drummond et al. 2012). After evaluating tree loglikelihood scores using Tracer (v1.6.0; Rambaut et al. 2014) we calculated the maximum clade credibility tree from 10000 trees with TreeAnnotator (v1.6.2). We coded each terminal branch by the biogeographic region from which it was found (or black for widespread lineages).

\section{RESULTS AND DISCUSSION}

\section{Biogeographic regional parasite diversity}

A total of 983 infections were reported in resident birds from sub-Saharan Africa. The Congolian region had 249 infections (71 Haemoproteus, 175 Plasmodium, 3 Leucocytozoon); the South African had 76 infections (19 Haemoproteus, 57 Plasmodium, 0 Leucocytozoon); the Sudanian had 167 infections (61 Haemoproteus, 62 Plasmodium, 44 Leucocytozoon); and the Zambezian had 491 infections (121 Haemoproteus, 216 Plasmodium, 154 Leucocytozoon). Focusing on lineages rather than infections, the number of lineages by genus found within only one region varied (chi-square, $P<0.0002$, D.F. $=6$ ); notably, when excluding lineages reported only once, the Congolian region harbours no Leucocytozoon lineages in resident birds. However, migratory birds collected there have been infected with this parasite genus (see Hellgren et al. 2007).

The different proportions of parasite genera between regions may be due to the ecological distributions of their vectors or to uneven sampling, or to a combination thereof. For example, Plasmodium and its Culex mosquito vectors (Garnham, 1966; Valkiūnas, 2005) tend to be broadly distributed (Clark et al. 2014), but Culex are limited by temperature and precipitation, and require standing water (often stagnant) for breeding (Patz and Olson, 2006; other mosquito genera utilize floodwater areas). The genus Haemoproteus and its subgenera Haemoproteus and Parahaemoproteus are vectored by several species of hippoboscid flies (Hippoboscidae) and biting midges (Culicoides), respectively (Atkinson and van Riper, 1991). Both hippoboscids and biting midges tend to range from semi-moist to more arid regions but require moist soil or water for breeding (Meiswinkel et al. 2004). Finally, Leucocytozoon is vectored by black flies (Simuliidae), which require clean, flowing water to lay their eggs (Carlsson, 1967). 
These different environmental preferences of vectors could explain some of the parasite genera proportions we found within regions. In the comparatively moist/humid Congolian region for example, Plasmodium dominated the infections (Fig. 1) with a moderate level of Haemoproteus, and Leucocytozoon was virtually absent. Importantly however, Leucocytozoon was found in many migrants from the Congolian region, the data for which were excluded from our analyses (see MalAvi; Bensch et al. 2009). This same pattern held for the Sudanian region, which seems anomalous given that region is far more arid than the Congolian. However the specific localities sampled in the Sudanian region were close to the Congolian region. Thus, the higher rates of infection by Plasmodium and Haemoproteus would be expected. The same was also true for the Zambezian, a region also drier than the Congolian. Given that the bulk of the Zambezian sampling comes from a single study focused on the Vwaza Marshes of Malawi (Lutz et al. 2015), the high levels of Plasmodium and Haemoproteus are also expected. We would anticipate that sampling in the Sudanian and Zambezian regions, away from extensive water sources, would result in lower regional prevalence of Plasmodium and Haemoproteus. Finally, the Southern African region (which has the poorest sampling to date; Fig. 1) is dominated by Plasmodium infections, with a lower level of Haemoproteus infection and no Leucocytozoon infections. Given the broad range of habitat variation across this region, particularly in South Africa, we would expect that more intensive and systematic sampling will yield not only higher levels of Haemoproteus and Leucocytozoon infections, but also strong intraregional differences in genus level infections.

\section{Geographic distribution of parasite lineages}

There was no phylogenetic structuring of lineages by biogeographic region (Fig. 2), despite the differences in broad scale distributions of parasites at the generic level (Fig. 1) and the high proportions of endemics in several regions. This is probably due to the predominantly-sampled widespread avian families (largely Passeriformes, Table 3) that would homogenize, to some extent, parasite distributions across regions (see below). If this limited structure holds up to further sampling, this pattern would indicate the importance of other factors such as vector distributions and connectivity between regions due to host movements.

\section{Host preferences}

With a few exceptions based on geographic distributions, passerine families are infected by Plasmodium more than they are by Haemoproteus

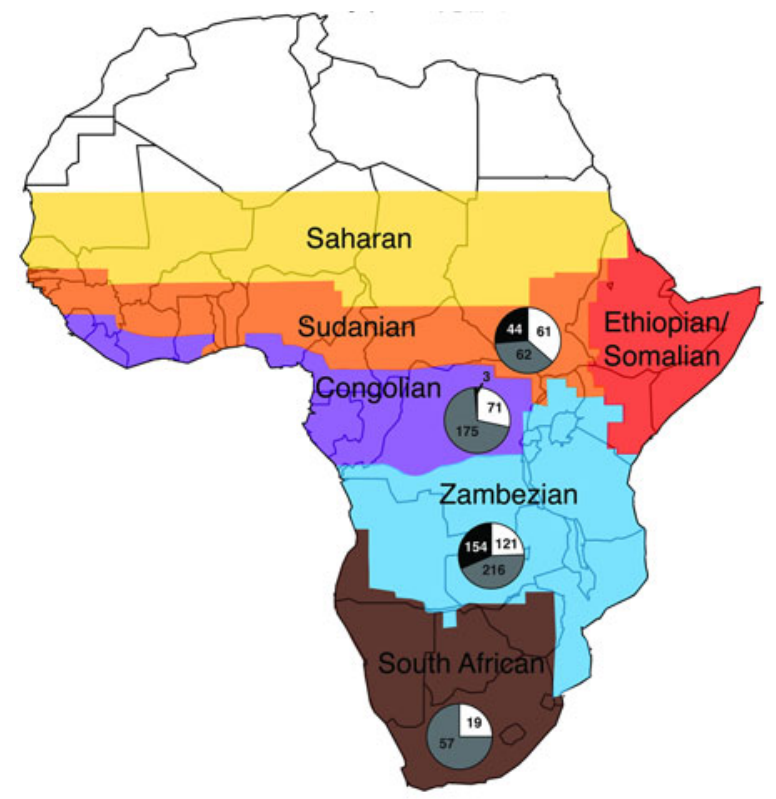

Fig. 1. Biogeographic regions of African avifauna (from Linder et al. 2012). Pie charts represent the relative proportions of parasite genera in each region (Grey: Plasmodium, Black: Leucocytozoon; White: Haemoproteus). Numbers in pie charts are the number of infections of each genus.

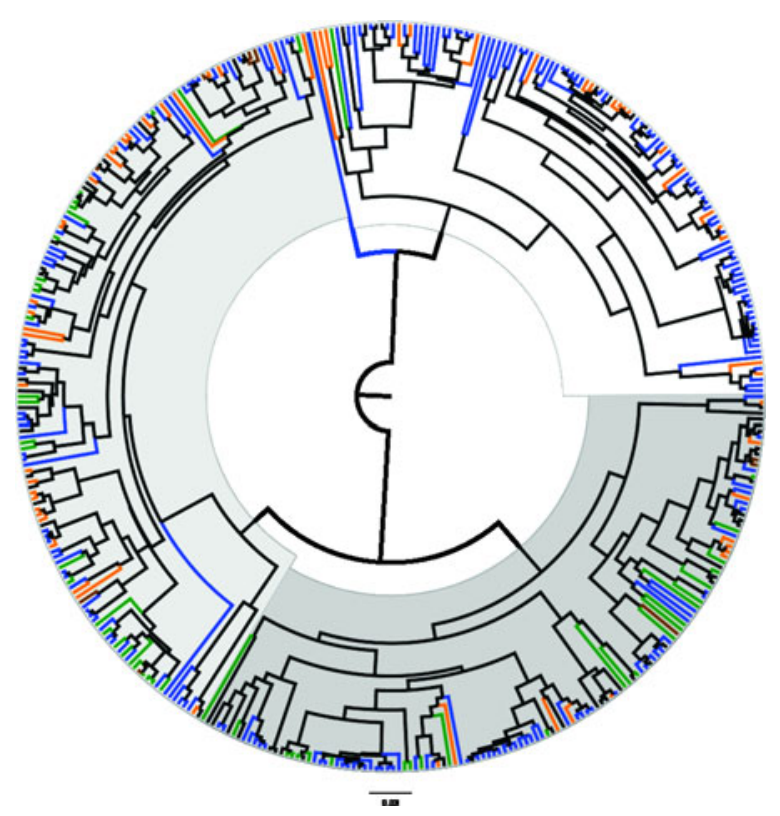

Fig. 2. Phylogenetic reconstruction African avian malaria parasites. Coloured terminal branches correspond to biogeographic regions: Congolian - purple, Southern African - brown, Sudanian - orange, Zambezian - blue, widespread - black. Clades are highlighted to genus: Plasmodium - light grey, Leucocytozoon - white, Haemoproteus - medium grey. Note that branch lengths are depicted as in a cladogram for simplification.

and Leucocytozoon (Table 3); however, very few studies sample all three genera at the same time, and these biases through 'lack of' sampling would almost certainly affect our interpretations. Weavers 
Table 3. Number of infections collected from hosts by host family, and number of lineages from each parasite genus by host family. Note that the number of lineages most often does not add up to the number of infected birds.

\begin{tabular}{|c|c|c|c|c|c|}
\hline \multirow[b]{2}{*}{ Host order } & \multirow[b]{2}{*}{ Host family } & \multirow[b]{2}{*}{$N$ infections } & \multicolumn{3}{|c|}{ Number of lineages } \\
\hline & & & Haemoproteus & Plasmodium & Leucocytozoon \\
\hline Falconiformes & Accipitridae & 5 & 1 & 4 & \\
\hline Passeriformes & Alaudidae & 3 & & 3 & \\
\hline Coraciiformes & Alcedinidae & 14 & 9 & 2 & 1 \\
\hline Anseriformes & Anatidae & 1 & & 1 & \\
\hline Ciconiiformes & Ardeidae & 2 & 1 & & \\
\hline Bucerotiformes & Bucerotidae & 3 & & 1 & 2 \\
\hline Cuculiformes & Centropidae & 2 & 1 & 1 & \\
\hline Passeriformes & Cisticolidae & 43 & 4 & 18 & 7 \\
\hline Coliiformes & Coliidae & 1 & & 1 & \\
\hline Columbiformes & Columbidae & 12 & 9 & 1 & 2 \\
\hline Coraciiformes & Coraciidae & 1 & & & 1 \\
\hline Passeriformes & Corvidae & 5 & 2 & 2 & 1 \\
\hline Cuculiformes & Cuculidae & 1 & 1 & & \\
\hline Passeriformes & Dicruridae & 4 & 1 & 3 & \\
\hline Passeriformes & Estrildidae & 55 & 11 & 17 & 11 \\
\hline Passeriformes & Eurylaimidae & 1 & & & 1 \\
\hline Passeriformes & Fringillidae & 27 & 7 & 13 & 5 \\
\hline Passeriformes & Hirundinidae & 4 & 1 & 3 & \\
\hline Passeriformes & Indicatoridae & 4 & 1 & 3 & \\
\hline Piciformes & Lybiidae & 4 & 4 & & \\
\hline Passeriformes & Malaconotidae & 15 & & 6 & 7 \\
\hline Coraciiformes & Meropidae & 5 & 4 & 2 & \\
\hline Passeriformes & Monarchidae & 8 & 2 & 3 & \\
\hline Passeriformes & Motacillidae & 9 & 2 & 2 & 3 \\
\hline Passeriformes & Muscicapidae & 68 & 11 & 24 & 17 \\
\hline Musophagiformes & Musophagidae & 1 & 1 & & \\
\hline Passeriformes & Nectariniidae & 123 & 21 & 30 & 11 \\
\hline Galliformes & Numididae & 2 & 2 & & \\
\hline Passeriformes & Paridae & 4 & & 2 & 2 \\
\hline Passeriformes & Passeridae & 16 & 6 & 8 & \\
\hline Galliformes & Phasianidae & 7 & 1 & 1 & 3 \\
\hline Piciformes & Picidae & 4 & 1 & 2 & 1 \\
\hline Passeriformes & Platysteiridae & 21 & 3 & 10 & 8 \\
\hline Passeriformes & Ploceidae & 116 & 29 & 21 & 24 \\
\hline Psittaciformes & Psittacidae & 1 & & 1 & \\
\hline Passeriformes & Pycnonotidae & 126 & 12 & 28 & 30 \\
\hline Gruiformes & Rallidae & 2 & & 2 & \\
\hline Ciconiiformes & Scopidae & 2 & 1 & & 1 \\
\hline Passeriformes & Stenostiridae & 6 & 1 & 2 & 3 \\
\hline Strigiformes & Strigidae & 5 & 3 & & 1 \\
\hline Passeriformes & Sturnidae & 17 & 8 & 5 & 3 \\
\hline Passeriformes & Sylviidae & 39 & 18 & 9 & 6 \\
\hline Passeriformes & Timaliidae & 23 & 11 & 6 & 4 \\
\hline Trogoniformes & Trogonidae & 3 & 1 & 1 & 1 \\
\hline Passeriformes & Turdidae & 74 & 9 & 22 & 14 \\
\hline Passeriformes & Zosteropidae & 15 & 6 & 3 & 4 \\
\hline
\end{tabular}

(Ploceidae) are infected by all three parasite genera, but slightly more by Haemoproteus. Old World warblers (Sylviidae) are also infected by all three parasite genera, but again, more so by Haemoproteus. Sampling of bush-shrikes (Malaconotidae) is low, but none are infected by Haemoproteus. Old World sparrows (Passeridae) are seemingly not infected by Leucocytozoon. Sampling of non-passerine families has been geographically spotty and taxonomically biased, and therefore we need much more broad sampling to firmly establish patterns of host use.
Previous studies have shown how variable parasite distributions can be, and for example, that the distribution of malaria parasites across the landscape was dependent on habitat (distance from rivers), or age and sex of the bird (Wood et al. 2007). The differing number of lineages parasitizing a host species or family may be due to migratory behaviour, habitat (even microhabitat as different vectors may specialize in different strata), other environmental variables, host evasion and other behaviours (gregariousness; Atkinson and van Riper, 1991; 
Table 4. Host specificity indices by parasite genus.

\begin{tabular}{llccc}
\hline \hline Genus & $n$ & $N$ of host species & $S_{\mathrm{TD}}$ & Var $S_{\mathrm{TD}}$ \\
\hline Haemoproteus & 110 & 84 & $4 \cdot 64$ & $0 \cdot 33$ \\
Leucocytozoon & 108 & 61 & $4 \cdot 12$ & $0 \cdot 00$ \\
Plasmodium & 298 & 161 & $4 \cdot 17$ & $0 \cdot 27$ \\
All & 516 & 202 & $4 \cdot 28$ & $0 \cdot 25$ \\
\hline \hline
\end{tabular}

Loiseau et al. 2010; Rifkin et al. 2012; GarciaLongoria et al. 2014; Olsson-Pons et al. 2015), as well as detection biases, i.e. abortive stages of development and PCR bias. For example, the somewhat higher levels of Haemoproteus infections in weavers could possibly be explained by the ease with which hippoboscid flies and midges could move between nests - many weaver species tend to nest in dense colonies. However, the former nesting behaviour is not characteristic of sylviids, which also showed higher Haemoproteus infections.

Parasite genera are not host-specific. Values of the Host Specificity Index $\left(S_{\mathrm{TD}}\right.$ ranged from $4 \cdot 17$ to $4 \cdot 64$; Table 4) reveal a low level of host specificity across all three parasite genera. When all the three genera are grouped the host specificity index remains high with a value of $4 \cdot 28$. Thus, parasite genera are widely distributed across Superorder and Orders of birds. VarSTD values were low, which indicates taxonomic structure was even across all parasite genera (individually and grouped) and further indicates that common ancestor distance of hosts were evenly distributed and reached at a high taxonomic level (in this case at the level of Order).

\section{FUTURE DIRECTIONS}

There are major sampling gaps of avian malaria parasites in Africa, and this is problematic for understanding a range of malaria-related topics, from simple assessments of lineage diversity to regional assemblages and endemism to broader ecological questions related to vector habitat preferences and how and why some lineages are broadly distributed while others are not (i.e. the AbundanceOccupancy Relationship; Drovetski et al. 2014; see also Lauron et al. 2015). Indeed, we find that when we sample a new region or a new host-taxonomic group, that we almost inevitably find new parasite lineages at both the species- and genus-level (Martinsen et al. 2016; Outlaw, unpublished data). The basic solution to all of these problems is simple: extensive geographic sampling is required and needs to include both molecular approaches and microscopy. And, sampling at each location should be as extensive as possible in terms of avian hosts (i.e. the broadest possible taxonomic diversity) and numbers of individual hosts sampled per species to convert singletons (or just a handful of birds of a given species) to reasonable frequencies for comparisons. Post-sampling, molecular assessments should be standardized in an effort to recover all the three malaria genera. Combined, these rather straightforward suggestions can provide an abundance of meaningful comparative data, even if just two regions are being compared (e.g. Mata et al. 2015).

Despite sampling issues (both taxonomic and molecular), currently available data from subSaharan Africa does suggest that there are a substantial number of endemic malaria lineages within avifaunal regions, and that this endemism may in turn be related to regional avian host endemism. Further, there does seem to be a general relationship between the type of habitat in which a host was sampled, and the prevalence of malaria parasite genera, as one would predict based on vector breeding-habitat preference, Plasmodium tends to be more prevalent in mesic habitats because mosquitoes require standing water, Haemoproteus tend to be more prevalent in xeric habitats because midges often only require moist soil, and Leucocytozoon is tied to running water, which its black fly hosts require for breeding. To extend our call for additional sampling, intra-regional microhabitats are another missing puzzle piece, as intra-regional dynamics may be as important and perhaps of more interest than coarse inter-regional studies. The Vwaza Marsh study by Lutz et al. (2015) is an excellent example of this. And, temporal variation may also play a role in malaria distribution in that temperature regimes may cause cyclical variation in prevalence; accounting for this should be considered, when and where possible (see SvenssonCoelho et al. 2013).

As we increase sampling across and within African bioregions, and indeed bioregions on any continent, we will be able to better decipher patterns of diversification across different scales (from interregional to micro-habitat). A broader knowledge of biogeographic patterns will not only help us better understand patterns and processes of malaria distributions, but will naturally lead to informative studies of vectors and how their natural history impacts malaria distribution. This knowledge can also provide insight into studies of why some avian hosts are more susceptible to infection, why some hosts are more likely to carry parasites between 
regions or continents, and why some host species tend to be more highly parasitized than others (see also Clark et al. 2014). An added benefit will be that the extensive sampling of avian hosts will allow for much finer scale phylogeographic/population genetic sampling of those hosts than is currently available for most species. This is particularly important in understory-dwelling host species found in Afrotropical forests, where recent work has uncovered extensive cryptic diversity (e.g, Huntley and Voelker, 2016). And together, extensive sampling of malaria parasites and their hosts will allow for powerful and meaningful assessments of the impacts of climate change over time. Ultimately, we do not know what is out there waiting to be discovered, and to expand our knowledge in these areas, it is all about the sampling.

\section{ACKNOWLEDGEMENTS}

This work would not have been possible without the samples that have been collected over the years across Africa, and the MalAvi database. We thank two anonymous reviewers whose critiques improved the manuscript. This is publication number 1532 of the Biodiversity Research and Teaching Collections at Texas A\&M.

\section{FINANCIAL SUPPORT}

This research received no specific grant from any funding agency, commercial or not-for-profit sectors.

\section{REFERENCES}

Atkinson, C. T. and van Riper, C., III (1991). Epizootiology and pathogenicity of avian hematozoa: Plasmodium, Haemoproteus, and Leucocytozoon. In Bird-Parasite Interactions. Ecology, Evolution and Behavior (ed. Loye, J. E. and Zuk, M.), pp. 19-48. Oxford University Press, New York.

Beadell, J. S., Ishtiaq, F., Covas, R., Melo, M., Warren, B. H., Atkinson, C. T., Bensch, S., Graves, G. R., Jhala, Y. V., Peirce, M. A., Rahmani, A. R., Fonseca, D. M. and Fleischer, R. C. (2006). Global phylogeographic limits of Hawaii's avian malaria. Proceedings of the Royal Society B: Biological Sciences 273, 2935-2944.

Beadell, J. S., Covas, R., Gebhard, C., Ishtiaq, F., Melo, M., Schmidt, B. K., Perkins, S. L., Graves, G. R. and Fleischer, R. C. (2009). Host associations and evolutionary relationships of avian blood parasites from West Africa. International Fournal for Parasitology 39, 257-266.

Bennett, G. F. and Herman, C. M. (1976). Blood parasites of some birds from Kenya, Tanzania and Zaire. Fournal of Wildlife Diseases 12, 59-65. Bennett, G. F., Blancou, J., White, E. M. and Williams, N. A. (1978). Blood parasites of some birds from Senegal. Journal of Wildlife Diseases 14, 67-73.

Bennett, G. F., Earle, R. A., Du Toit, H. and Huchzermeyer, F. W. (1992). A host-parasite catalogue of the haematozoa of the sub-Saharan birds. Onderstepoort Fournal of Veterinary Research 59, 1-73.

Bensch, S., Hellgren, O. and Pérez-Tris, J. (2009). MalAvi: a public database of malaria parasites and related haemosporidians in avian hosts based on mitochondrial cytochrome $b$ lineages. Molecular Ecology Resources 5, 1353-1358.

Bonneaud, C., Sepil, I., Milá, B., Buermann, W., Pollinger, J., Sehgal, R., Valkiūnas, G., Iezhova, T., Saatchi, S. and Smith, T. B. (2009). The prevalence of avian Plasmodium is higher in undisturbed tropical forests of Cameroon. Fournal of Tropical Ecology 25, 439-447.

Carlsson, G. (1967). Environmental factors influencing blackfly populations. Bulletin of the World Health Organization 37, 139.
Chasar, A., Loiseau, C., Valkiūnas, G., Iezhova, T., Smith, T. B. and Sehgal, R. (2009). Prevalence and diversity patterns of African avian blood parasites in degraded habitats. Molecular Ecology 18, 4121-4133.

Clark, N. J., Clegg, S. M. and Lima, M. R. (2014). A review of global diversity in avian Haemosporidians (Plasmodium and Haemoproteus: Haemosporida): new insights from molecular data. International Fournal for Parasitology 44, 329-338.

Clarke, K. R. and Warwick, R. M. (2001). A further biodiversity index applicable to species lists: variation in taxonomic distinctness. Marine Ecology Progress Series 216, 265-278.

Dickinson, E. C. and Remsen, J. V., Jr. (2013). The Howard and Moore Complete Checklist of the Birds of the World. No. Ed. 4. Aves Press, Eastborn, UK.

Drovetski, S. V., Aghayan, S. A., Mata, V. A., Lopes, R. J., Mode, N. A., Harvey, J. A. and Voelker, G. (2014). Does the niche-breadth or trade-off hypothesis explain the abundance-occupancy relationship in avian haemosporidia? Molecular Ecology 23, 3322-3329.

Drummond, A. J., Suchard, M. A., Xie, D. and Rambaut, A. (2012). Bayesian phylogenetics with BEAUti and the BEAST 1.7. Molecular Biology and Evolution 29, 1969-1973.

Durrant, K. L., Reed, J. L., Jones, P. J., Dallimer, M., Cheke, R. A., McWilliam, A. N. and Fleischer, R. C. (2007). Variation in haematozoan parasitism at local and landscape levels in the red-billed quelea Quelea quelea. Fournal of Avian Biology 38, 662-671.

Fjeldså, J. (2003). Patterns of endemism in African birds: how much does taxonomy matter? Ostrich 74, 30-38.

Garcia-Longoria, L., Garamszegi, L. Z. and Moller, A. P. (2014). Host escape behavior and blood parasite infections in birds. Behavioral Ecology 1-11.

Garnham, P. (1950). Blood parasites of east African vertebrates, with a brief description of exo-erythrocytic schizogony of $P$. pitmani. Parasitology 40, 328-337.

Garnham, P. (1966). Malaria Parasites and Other Haemosporidia. Blackwell Scientific Publications, Oxford University Press, New York.

Hellgren, O., Waldenström, J., Peréz-Tris, J., Szöll, E., Si, Ö., Hasselquist, D., Krizanauskiene, A., Ottosson, U. and Bensch, S. (2007). Detecting shifts of transmission areas in avian blood parasites - a phylogenetic approach. Molecular Ecology 16, 1281-1290.

Huntley, J. W. and Voelker, G. (2016). Cryptic diversity in Afro-tropical forests: the systematics and biogeography of the avian genus Bleda. Molecular Phylogenetics and Evolution 99, 297-308.

Iezhova, T.A., Dodge, M., Sehgal, R. N.M., Smith, T.B. and Valkiūnas, G. (2011). New avian Haemoproteus species (Haemosporida: Haemoproteidae) from African birds, with a critique of the use of host taxonomic information in hemoproteid classification. Fournal of Parasitology 97, 682-694.

Ishak, H. D., Dumbacher, J.P., Anderson, N. L., Keane, J. J., Valkiünas, G., Haig, S. M., Tell, L. A., and Sehgal, R. N. M. (2008). Blood parasites in Owls with conservation implications for the Spotted Owl (Strix occidentalis). PLoS ONE 3, e2304.

Ishtiaq, F., Beadell, J. S., H.warren, B. and Fleischer, R. C. (2012). Diversity and distribution of avian haematozoan parasites in the western Indian Ocean region: a molecular survey. Parasitology 139, 221-231.

Jetz, W. and Rahbek, C. (2002). Geographic range size and determinants of avian species richness. Science 297, 1548-1551.

Karamba, K. I., Kawo, A. H., Dabo, N. T. and Mukhtar, M. D. (2012) A survey or avian malaria parasite (sic) in Kano State, Northern Nigeria. International fournal for Biotechnology and Molecular Biology Research 3, $8-14$.

Kirkpatrick, C.E. and Smith, T. B. (1988). Blood parasites of birds in Cameroon. Fournal of Parasitology 74, 1009-1013.

Lauron, E. J., Loiseau, C., Bowie, R. C. K., Spicer, G. S., Smith, T. B., Melo, M. and Sehgal, R. N.M. (2015). Coevolutionary patterns and diversification of avian malaria parasites in African sunbirds (Family Nectariniidae). Parasitology 142, 635-647.

Linder, H. P., de Klerk, H. M., Born, J., Burgess, N. D., Fjeldså, J. and Rahbek, C. (2012). The partitioning of Africa: statistically defined biogeographical regions in sub-Saharan Africa. Fournal of Biogeography 39, 11891205.

Loiseau, C., Valkiūnas, G., Chasar, A., Hutchinson, A., Iezhova, T. and Sehgal, R. N. M. (2010). Spatial variation of haemosporidian parasite infection in African rainforest bird species. Fournal of Parasitology 96, $21-29$

Loiseau, C., Harrigan, R. J., Robert, A., Bowie, R. C. K., Thomassen, A., Smith, T. B. and Sehgal, R. (2012). Host and habitat specialization of avian malaria in Africa. Molecular Ecology 21, 431-441. 
Lutz, H. L., Hochachka, W. M., Engel, J. I., Bell, J. A., Tkach, V. V., Bates, J. M., Hackett, S. J. and Weckstein, J. D. (2015). Parasite prevalence corresponds to host life history in a diverse assemblage of afrotropical birds and haemosporidian parasites. PLoS ONE 10(4), e0121254.

Martinsen, M. S., Mcinerney, N., Brightman, H., Ferebee, K. Walsh, T., Mcshea, W. J., Forrester, T. D., Ware, L., Joyner, P. H., Perkins, S. L., Latch, E. K., Yabsley, M. J., Schall, J. J. and Fleischer, R. C. (2016). Hidden in plain sight: cryptic and endemic malaria parasites in North American white-tailed deer (Odocoileus virginianus). Science Advances.

Mata, V. A., da Silva, L. P., Lopes, R. J. and Drovetski, S. V. (2015) The Strait of Gibraltar poses an effective barrier to host-specialised but not to host-generalised lineages of avian Haemosporidia. International Fournal of Parasitology 45, 711-719.

Meiswinkel, R., Venter, G. J. and Nevill, E. M. (2004). Vectors: Culicoides Spp. Infectious Diseases of Livestock. Oxford University Press, Cape Town, pp. 93-136.

Møller, A.P., Garamszegi, L. Z., Peralta-Sánchez, J. M. and Soler, J. J. (2011). Migratory divides and their consequences for dispersal, population size and parasite-host interactions. Fournal of Evolutionary Biology 24, 1744-1755.

Okanga, S., Cumming, G.S., Hockey, P.A.R., Nupen, L. and Peters, J. L. (2014). Host specificity and co-speciation in avian Haemosporidia in the Western Cape, South Africa. PLoS ONE 9(2), e86382.

Olsson-Pons, S., Clark, N. J., Ishtiaq, F. and Clegg, S. M. (2015) Differences in host species relationships and biogeographic influences produce contrasting patterns of prevalence, community composition and genetic structure in two genera of avian malaria parasites in southern Melanesia. Fournal of Animal Ecology 84, 985-998.

Patz, J. A. and Olson, S. H. (2006). Malaria risk and temperature: influences from global climate change and local land use practices. Proceedings of the National Academy of Sciences of the United States of America 103, 5635-5636.

Pérez-Tris, J., Hellgren, O., Križanauskiene, A., Waldenström, J., Secondi, J., Bonneaud, C., Fjeldså, J., Hasselquist, D. and Bensch, S. (2007). Within-host speciation of malaria parasites. PLoS $O N E 2$, e235.

Poulin, R. and Mouillot, D. (2003). Parasite specialization from a phylogenetic perspective: a new index of host specificity. Parasitology 126, 473-480.

Rambaut, A., Suchard, M. A., Xie, D. and Drummond, A. J. (2014).

Tracer v1.6. http://tree.bio.ed.ac.uk/software/tracer/.
Ricklefs, R. E. and Fallon, S. M. (2002). Diversification and host switching in avian malaria parasites. Proceedings of the Royal Society B: Biological Sciences 269, 885-892.

Ricklefs, R. E., Outlaw, D. C., Coelho, M. S., Medeiros, M., Ellis, V. A. and Latta, S. (2014). Species formation by host switching in avian malaria parasites. Proceedings of the National Academy of Sciences of the United States of America 111, 14816-14821.

Rifkin, J. L., Nunn, C. L. and Garamszegi, L.Z. (2012). Do animals living in larger groups experience greater parasitism? A meta-analysis. American Naturalist 180, 70-82.

Santiago-Alarcon, D., Palinauskas, V. and Schaefer, H. M. (2012). Diptera vectors of avian Haemosporidian parasites: untangling parasite life cycles and their taxonomy. Biological Reviews 87, 928-964.

Sehgal, R. N., Jones, H. I. and Smith, T. B. (2005). Blood parasites of some West African rainforest birds. Fournal of Veterinary Medical Science 67, 295-301.

Sinclair, I. and Ryan, P. (2010). Birds of Africa, South of the Sahara. Struik Nature, Cape Town.

Smith, T. B., H. Thomassen, A., Freedman, A. H., Sehgal, R. Buermann, W., Saatchi, J., Pollinger, S., Milá, B., Pires, D. Valkiūnas, G. and Wayne, R. K. (2011). Patterns of divergence in the olive sunbird (Cyanomitra olivacea) across the African rainforest-savanna ecotone. Biological Fournal of the Linnean Society 103, 825-835.

Svensson-Coelho, M., Blake, J. G., Loiselle, B. A., Penrose, A. S., Parker, P. G. and Ricklefs, R. E. (2013). Diversity, prevalence, and host specificity of avian Plasmodium and Haemoproteus in a western Amazon assemblage. Ornithological Monographs 76, 1-47.

Valkiūnas, G. (2005). Avian Malaria Parasites and Other Haemosporidia. CRC Press, Boca Raton.

Valkiūnas, G., Sehgal, R. N., Iezhova, T. A. and Smith, T. B. (2005). Further observations on the blood parasites of birds in Uganda. Fournal of Wildlife Diseases 41, 580-587.

Valkiūnas, G., Iezhova, T. A., Loiseau, C., Smith, T. B. and Sehgal, R. N. M. (2009). New malaria parasites of the subgenus Novyella in African rainforest birds, with remarks on their high prevalence, classification and diagnostics. Parasitology Research 104, 1061-1077.

Waldenström, J., Bensch, S., Kiboi, S., Hasselquist, D. and Ottosson, U. (2002). Cross-species infection of blood parasites between resident and migratory songbirds in Africa. Molecular Ecology 11, 1545-1554. Wood, M. J., Cosgrove, C. L., Wilkin, T. A., Knowles, S. C. L., Day, K. P., and Sheldon, B. C. (2007). Within-population variation in prevalence and lineage distribution of avian malaria in blue tits, Cyanistes caeruleus. Molecular Ecology 16, 3263-3273. 